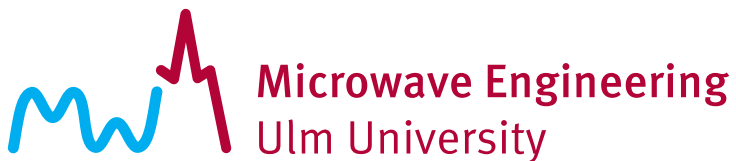

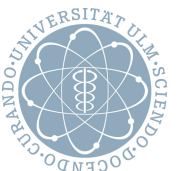

\section{Blind Adaptive Beamforming for Automotive Radar Interference Suppression}

Jonathan Bechter, Amarilda Demirlika, Philipp Hügler, Fabian Roos, and Christian Waldschmidt

(c) 2018 IEEE. Personal use of this material is permitted. Permission from IEEE must be obtained for all other uses, in any current or future media, including reprinting/republishing this material for advertising or promotional purposes, creating new collective works, for resale or redistribution to servers or lists, or reuse of any copyrighted component of this work in other works. 


\title{
Blind Adaptive Beamforming for Automotive Radar Interference Suppression
}

\author{
Jonathan Bechter, Amarilda Demirlika, Philipp Hügler, \\ Fabian Roos, and Christian Waldschmidt
}

\author{
Institute of Microwave Engineering \\ Ulm University, Germany \\ email: jonathan.bechter(at)alumni.uni-ulm.de
}

\begin{abstract}
Radar sensors offer enormous advantages as sensing devices for automated and autonomous driving. However, when multiple of such sensors are operated in a large number of cars, there is a high risk of the occurrence of mutual interferences. In the currently widespread linearly frequency modulated sensors these interferences reduce the detection performance, especially for targets with a low radar cross section. In this paper the interference effects are suppressed with an adaptive beamforming scheme based on a mean square error minimization. The paper evaluates the algorithm with the help of a simulated and measured scenario, and discusses the occurring interference effects and the benefits of the beamforming approach.
\end{abstract}

\section{Introduction}

Radar systems operating in the range of 76 to $81 \mathrm{GHz}$ become a common sensor device for automated and autonomous driving applications. There are challenging demands to the sensors - a wide field of view of up to $360^{\circ}$, high resolution in range, velocity and azimuth, and fast update rates. To fulfill those requirements it is necessary to transmit over a wide bandwidth for a long measurement duration, and to mount multiple sensors on a single vehicle. This leads to a nearly ubiquitous transmission of electromagnetic waves in a large part of the available frequency band.

As there is currently no regulation for the simultaneous operation of multiple radar sensors at the same time, the occurrence of mutual interferences is very likely. This issue is discussed often with focus on linearly frequency modulated sensors, because they are the most common radar sensors for automotive applications at the moment. Between this kind of sensors interferences typically cause time-limited disturbances of the baseband signals [1,2]. As a consequence there is an increase of the receiver noise level, e.g. as demonstrated in [3]. The increased noise level is especially critical for the detection of distant objects, or targets with a low radar cross section like pedestrians or bicycles.

In order to avoid this degradation of sensor performance it is necessary to implement countermeasures which compensate the interference effects. In this work, we address the interference problem with adaptive digital beamforming (ADBF). Beamforming in general alters the signal 


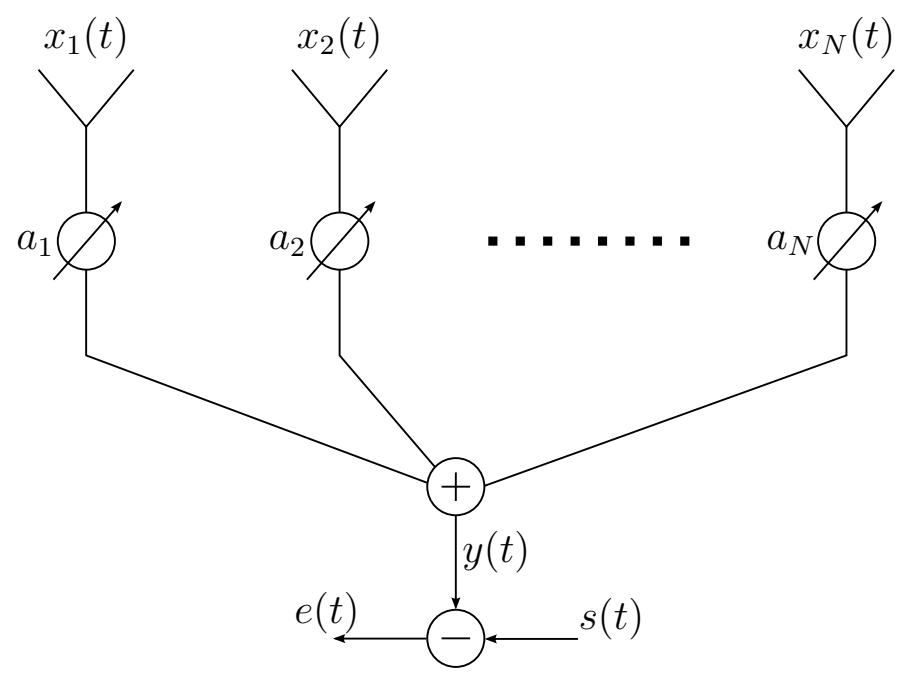

Figure 1: Setup of an adaptive beamformer following the MSE approach.

phase and amplitude between different antennas to create constructive or destructive superpositions of a signal depending on its incident angle. Therefore, an adaptive beamformer makes use of the input data statistics [4]. The adaptive processing offers the advantage, that target directions are taken into account without their exact knowledge, but by solving an optimization problem instead. Furthermore, the application of beamforming requires that all considered objects are in the far field of the antenna array, so that the well-known relation

$$
\Delta \varphi=k d \sin \vartheta
$$

holds for the phase difference of a signal between two antenna elements. The variables in the equation are wave number $k$, antenna element distance $d$, and incident angle of the signal $\vartheta$.

The beamforming that is described here shall improve the detection performance without disturbing the normal processing steps. In the interference-free case a range-Doppler matrix is calculated for each channel, followed by a non-coherent integration of all channels. This allows a good omni-directional detection performance preposed to a direction of arrival estimation. In the presence of interference the non-coherent integration is enhanced by the ADBF as described in Section 2. The presented scheme requires a detection of the interference in the baseband time signal, what is also addressed in this section. The ADBF scheme is then applied on simulation and measurement data in Section 3. Finally, the main results are summarized and the properties of the adaptive beamformer are discussed in a conclusion.

\section{Adaptive Beamforming Approach}

Adaptive beamforming solves an optimization criterion to achieve the complex-valued weights for the superposition of the signals at different antennas. Here, an MSE (Mean Square Error) minimization is used as derived in [5]. The general procedure is depicted in Fig. 1. The receive signal at the different antennas is summarized in the vector $\mathbf{x}(t)=\left[x_{1}(t) x_{2}(t) \ldots x_{N}(t)\right]^{T}$. The 
signals $\mathbf{x}(t)$ are multiplied with the weight vector $\mathbf{a}=\left[a_{1} a_{2} \ldots a_{N}\right]$ and added up to calculate the beamformer output $y(t)$. Under the assumption of a desired reference signal $s(t)$, it is possible to calculate the MSE as

$$
e^{2}(t)=|s(t)-y(t)|^{2}
$$

An optimum weight vector $\mathbf{a}_{\text {opt }}$ as a solution to this optimization problem is provided by the Wiener-Hopf equation [6, 7]:

$$
\mathbf{a}_{\mathrm{opt}}=\left(\mathbf{R}^{-1} \mathbf{p}\right)^{*}
$$

with the correlation matrix

$$
\mathbf{R}=\frac{1}{T} \sum_{t \in T} \mathbf{x}(t) \mathbf{x}(t)^{H} \in C^{N \times N}
$$

and the cross correlation vector

$$
\mathbf{p}=\frac{1}{T} \sum_{t \in T} s(t) \mathbf{x}(t) .
$$

The exponent $(\cdot)^{H}$ describes hermitian, and the exponent $(\cdot)^{*}$ describes complex conjugate. Small bold letters describe vectors, while the capital bold letter describes a matrix. The absolute of the vector $\mathbf{a}_{\text {opt }}$ is typically normalized to 1 . As a time duration $T$ for the calculation of $\mathbf{R}$ and $\mathbf{p}$ we use around 100 samples.

To determine $\mathbf{p}$ it is required to know the reference signal and the input signal at the same time instances. In general, this is not the case. However, in automotive radar applications it is possible to make use of the time limitation of the interfering signals in the baseband. This allows achieving a reference and an interfered input signal at the same time instances following the scheme depicted in Fig. 2. A block of samples of the interference-free signal part - of a single channel or the superposition of multiple channels - is used as a reference signal $s(t)$. The interfered part of the signal is added to $s(t)$ at an arbitrary time span, what results in an interfered input signal $\mathbf{x}(t)$ for each channel. Although the interference in $\mathbf{x}(t)$ is not present at each time instance, the signals $s(t)$ and $\mathbf{x}(t)$ can be compared to determine the solution of (3).

The interfered part that is added in order to create $\mathbf{x}(t)$ also contains the desired signal components, which will lead to small distortions. The influence of those distortions is reduced, when $\mathbf{x}(t)$ has a long duration in comparison to the number of interfered samples. However, this also reduces the interference suppression of the beamformer.

Please note that it is important to solve (3) with complex-valued signals when a chirp-sequence radar is considered. Otherwise, the adaptive beamformer will take into account the positive and negative frequency components of the typically real-valued receive signals. However, only one side of the frequency spectrum is interesting for the further signal processing. A Hilbert transform, as it is performed in [8], solves this problem. 


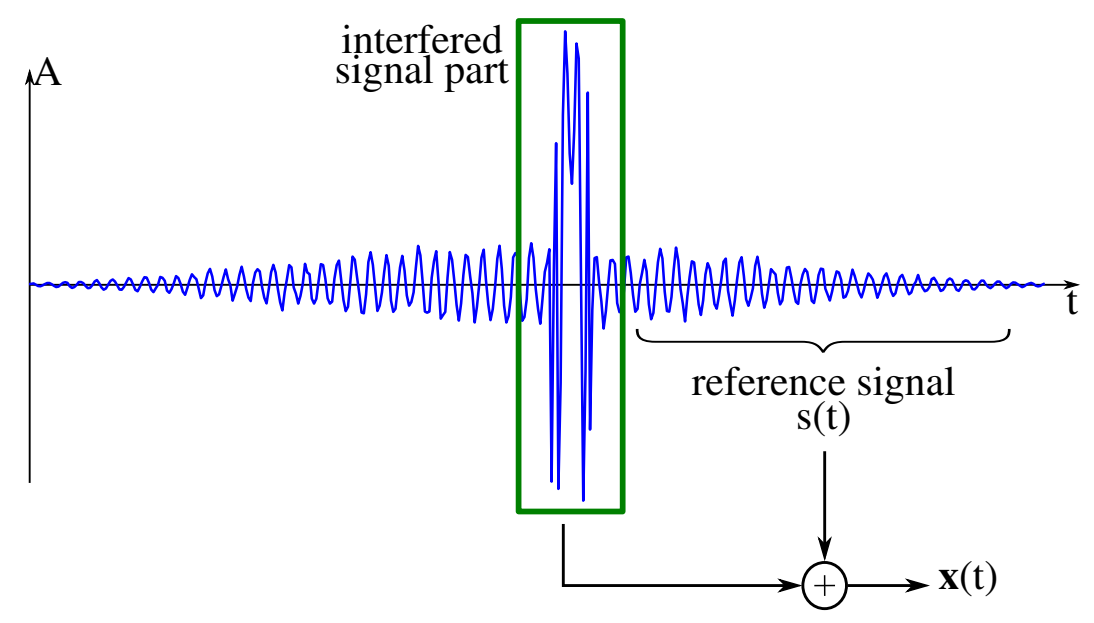

Figure 2: The time-limitation of the interference offers the possibility to acquire a desired signal $s(t)$ and an interfered signal $\mathbf{x}(t)$.

The described processing requires a detection of the interfered part of the time signal. There exist several methods for interference detection for linearly frequency modulated automotive radars. Most of them are based on a power detection, making use of the time-limited high-power spikes, e.g. a simple power detector as described in [9], or the more complicated detection scheme derived in [10] related to image processing. An interference detection based on the chirp-like frequency in the baseband is also possible, as demonstrated in [11].

Furthermore, it is not necessary to combine all $N$ channels of the radar system with the adaptive beamformer. The ADBF will not increase the power of targets that are present in the scene, as this does not optimize the MSE. Thus, it is advantageous to add up only a few channels with the ADBF in order to suppress the interference. Afterwards the multiple ADBF outputs are integrated non-coherently, as shown in Fig. 4. After this non-coherent integration a target detection, a direction of arrival (DoA) estimation, and any further processing steps can be performed.

\section{Simulation and Measurement}

For the evaluation of the described method, we consider a $76 \mathrm{GHz}$ multiple-input multipleoutput system with 3 transmit and 8 receive antennas, resulting in a 24 elements virtual uniform linear array with $\lambda / 2$-spacing. The front-end is depicted in Fig. 3. The radar uses a chirpsequence modulation with the parameters given in Tab. 1. The MIMO operation is achieved by a time-multiplexing of the transmitters, so the unambiguous velocity is reduced. However, we consider static scenarios only, so this issue does not influence the investigation. For both simulation and measurement four objects are located at the positions given in Tab. 2, whereby the last object also takes the role of an interference source. With these parameters all objects are located in the far field of the array and thus digital beamforming can be applied. It is carried out in accordance with the scheme in Fig. 4, so that bundles of 4 virtual antennas are selected for interference suppression with the adaptive beamforming. The six beamforming outputs are afterwards integrated non-coherently for an improved detection performance. This is done by 


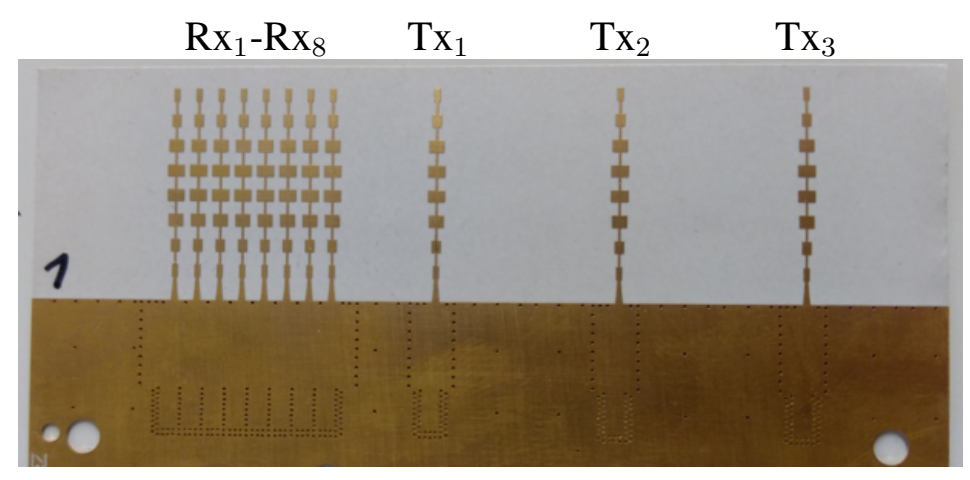

Figure 3: The antenna front-end used for the measurements consists of 3 transmit $\left(\mathrm{Tx}_{1}-\mathrm{Tx}_{3}\right)$ and 8 receive $\left(\mathrm{Rx}_{1}\right.$ $\left.\mathrm{Rx}_{8}\right)$ antennas.

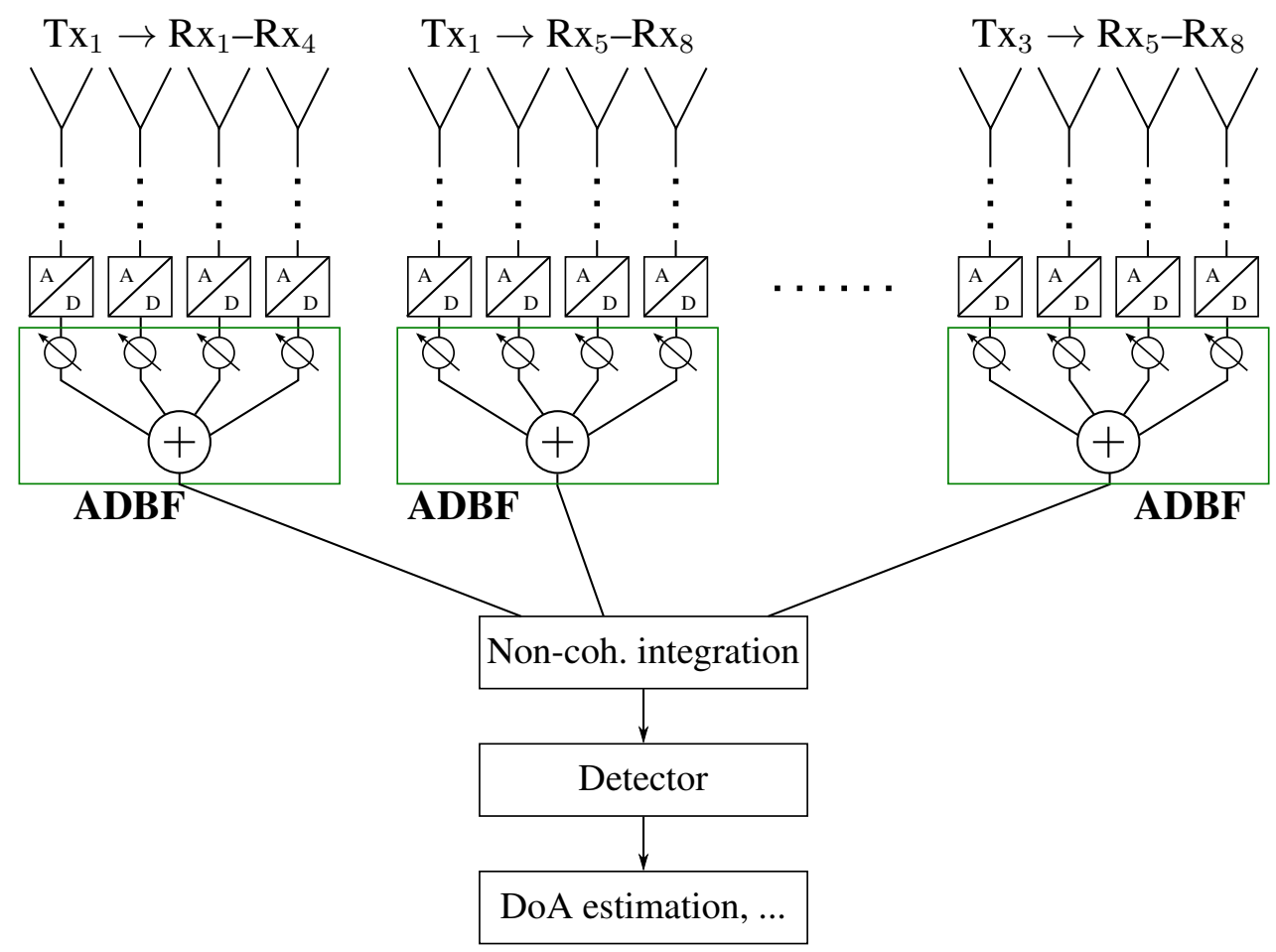

Figure 4: The response of each 4 virtual antennas is used to suppress the interference with the adaptive beamforming, followed by a non-coherent integration and further processing steps.

adding their absolutes and dividing the result by 6 .

The simulation considers an interferer with the same transmit power and antenna gain as the interfered sensor, which transmits a continuous wave signal. Both sensors have uncorrelated oscillators, what is realized in a simplified way by adding random start phases to the interfering signal at each frequency chirp. The receive powers of desired signals and interfering signal are calculated with the radar equation and the Friis equation, respectively.

The interference leads to a wide-band noise increase in the whole range-Doppler spectrum. Fig. 5 shows a single Doppler cell in the interference-free case, under the influence of interference, and after ADBF is applied. A comparison of interference-free and interfered signal shows 


\begin{tabular}{l|c} 
Parameter & Value \\
\hline Center frequency $f_{c}$ & $76 \mathrm{GHz}$ \\
Bandwidth $B$ & $500 \mathrm{MHz}$ \\
Ramp duration $T$ & $60 \mu \mathrm{s}$ \\
Ramp repetition rate $T_{\mathrm{r} 2 \mathrm{r}}$ & $70 \mu \mathrm{s}$ \\
Number Tx & 3 \\
Number Rx & 8 \\
Transmitted chirps & $3 \cdot 64$
\end{tabular}

Table 1: Modulation parameters for simulation and measurement.

\begin{tabular}{l|c|c|c} 
& $r$ & $\vartheta$ & $v$ \\
\hline Object 1 & $2.5 \mathrm{~m}$ & $-33.6^{\circ}$ & 0 \\
Object 2 & $3.11 \mathrm{~m}$ & $42.4^{\circ}$ & 0 \\
Object 3 & $4.87 \mathrm{~m}$ & $30.1^{\circ}$ & 0 \\
Object 4 (interferer) & $6.0 \mathrm{~m}$ & $10.1^{\circ}$ & 0
\end{tabular}

Table 2: Target properties for simulation and measurement.

a noise increase in the order of $30 \mathrm{~dB}$. This additional noise floor vanishes after the ADBF is applied. However, the target corresponding to the interferer disappears as well.

The effect of the beamforming is also visible in the beam pattern in Fig. 6, which is calculated based on the vector $\mathbf{a}_{\text {opt }}$ for four virtual antennas. The pattern shows a clear notch towards the interference direction $10^{\circ}$. The figure also contains a second pattern, which is calculated in the same way; however, the interferer amplitude in the time signal is reduced by a factor of 10 . This leads to a weaker suppression of the interfering signal at $10^{\circ}$. The interfered time signals are compared in Fig. 7a.

This behavior is interpreted as follows: when the interfering signal has a high amplitude, it dominates the MSE optimization criterion and the beamformer spends its main effort on interference suppression; when the amplitude is lower, the MSE is also influenced significantly by the target at $10^{\circ}$, so the ADBF chooses a trade-off between interference suppression and target conservation, as it is visible in the pattern.

The above scenario is also measured in an anechoic chamber. Interference is again created with a continuous wave signal at $76 \mathrm{GHz}$. A comparison of the interference-free range-Doppler spectrum in Fig. 8a and the interfered spectrum in Fig. 8b shows, that the interference turns out as an increased noise floor affecting only a few Doppler cells. In successive measurements the 2 stripes were shifted into other Doppler cells (not depicted in the paper), however they were always symmetric to $v=0$. We expect that the signal sources are sufficiently stable within a single measurement in such a way that there is a deterministic phase relation between the frequency ramps. Between two successive measurements the frequency difference of the oscillators drifts slightly, so that different Doppler cells are affected. The least stable reference oscillator we used in this setup had a stability of $20 \mathrm{ppm}$ at $80 \mathrm{MHz}$. 


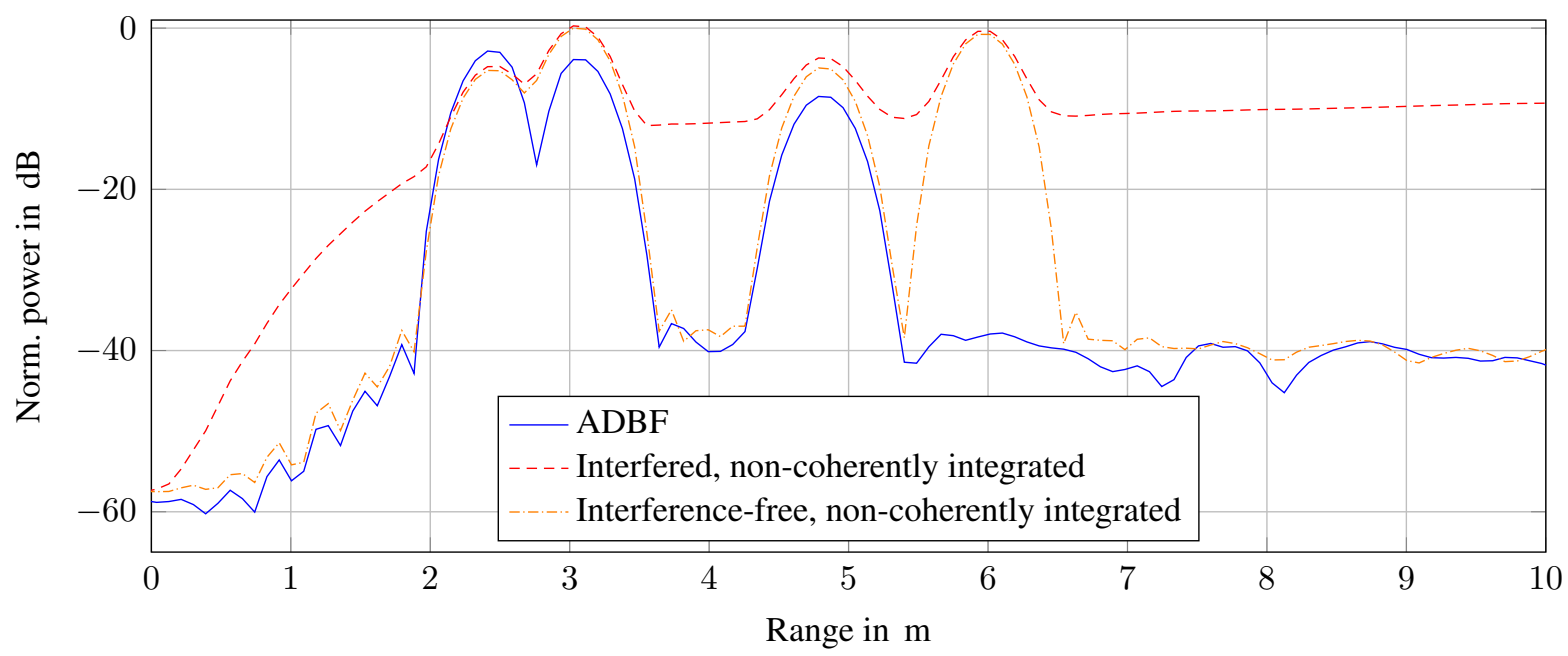

Figure 5: Comparison of simulated interference-free signal, interfered signal, and the result of the ADBF. The target acting as interferer is located at $6 \mathrm{~m}$. The plots are normalized to the maximum of the interference-free curve.

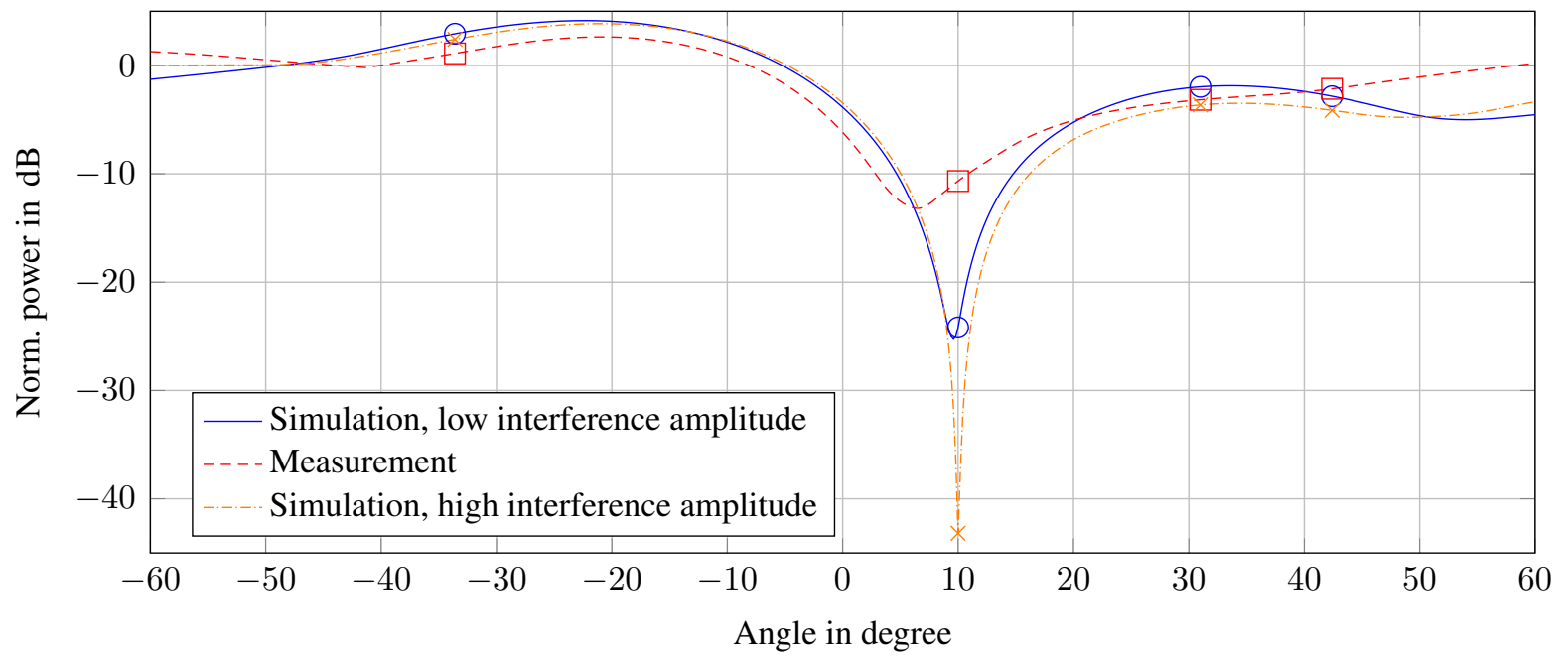

Figure 6: Beam pattern for the combination of 4 virtual antennas for different interference powers. The directions of targets are highlighted in the curves.

Nevertheless, the interference prevents a reliable detection in the affected Doppler cells, and - without foreknowledge - it is not possible to tell if targets are present at these velocities. The ADBF is applied to suppress the interference effects, which results in the range-Doppler spectrum in Fig. 8c. Most of the increased noise floor vanishes, but there are still some artifacts left in the spectrum, especially at the velocity $-6 \mathrm{~m} / \mathrm{s}$. The target related to the interferer itself is suppressed by only $6.6 \mathrm{~dB}$, so it is still detectable. The interfered time signal of a single frequency chirp in Fig. $7 b$ - integrated non-coherently over the signal of 8 receive antennas shows that the interference has a lower power compared to the simulation. As discussed in the end of the simulation part, this leads to a lower interference suppression. This is also visible from the respective curve in the beam pattern in Fig. 6. 


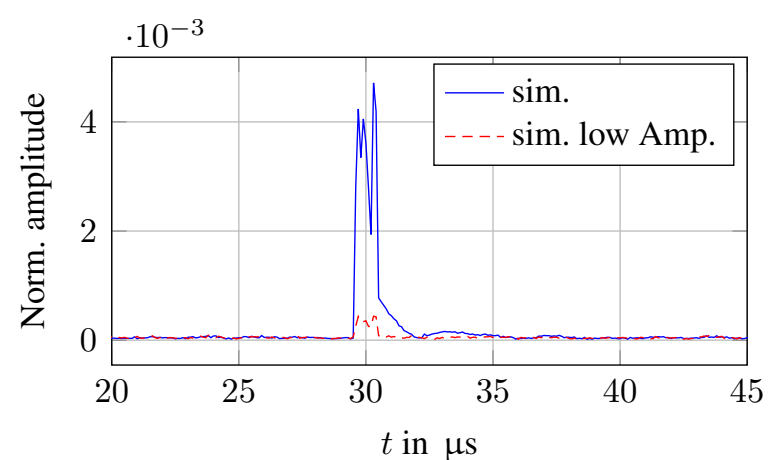

(a) Simulation, interference occurs between $28-32 \mu \mathrm{s}$.

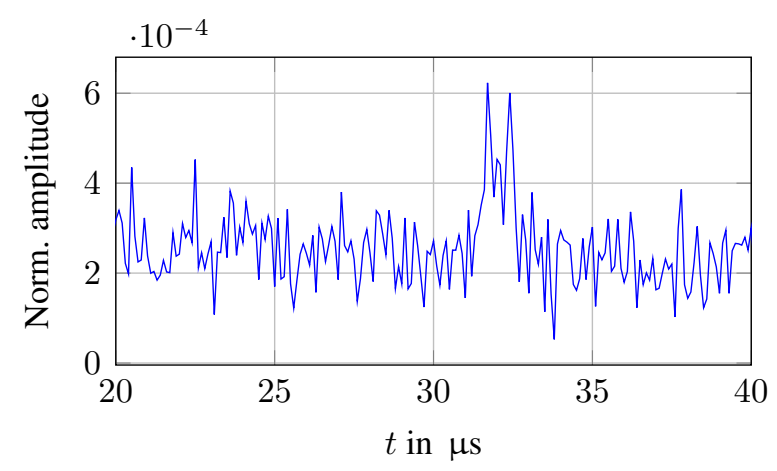

(b) Measurement, interference occurs between 30-35 $\mu$ s.

Figure 7: Comparison of interfered time domain signals, integrated non-coherently over 8 receive antennas for a single frequency ramp. The simulation is once performed with an interference amplitude calculated with the Friis equation, and once with a 10 times lower interference amplitude. The interfering signal in the measurement had a lower transmit power, visible on the right-hand side.

As a last point we discuss the remaining interference at the velocity $-6 \mathrm{~m} / \mathrm{s}$ after ADBF. Therefore, we consider a DoA estimation for both interfered velocity cells, $\pm 6 \mathrm{~m} / \mathrm{s}$. The estimation is performed with the signals transmitted by $\mathrm{Tx}_{1}$ only and should yield the direction of the interfering signal, $10^{\circ}$. This is happening in the case of $6 \mathrm{~m} / \mathrm{s}$, see Fig. $9 \mathrm{a}(-)$. However, for $-6 \mathrm{~m} / \mathrm{s}$ the power is not clearly focused towards a single DoA, as visible in Fig. 9b (-). This is happening because of the image frequency problem discussed in [12]: the receiver is limited to real valued signals, so that the power of interfering signals is distributed towards multiple DoAs. For further clarification we calibrate the received data with the complex conjugate phases and again perform a DoA estimation. This leads to the (---) curves in Fig. 9. Now there is a maximum at the inverse interferer DoA $-10^{\circ}$ in Fig. 9b, and a corrupted estimation in Fig. 9a. This is exactly what is expected from [12].

It is found from Fig. $9 \mathrm{~b}$ that the major part of the interference in the velocity cell $-6 \mathrm{~m} / \mathrm{s}$ is focused towards $42^{\circ}$, what is also the direction of a target. For MSE optimization the ADBF cannot suppress this target, so it does not fully suppress the respective interference component either. Thus, part of the interference energy remains in the range-Doppler spectrum.

\section{Conclusion}

Interference effects between automotive radars can degrade the detection performance, what is counteracted with adaptive digital beamforming. Because of the MSE optimization criterion, the described approach suppresses interferences proportional to their signal power and finds a trade-off between desired signals and interference. There is no foreknowledge of their directions required. However, targets with a large signal power are taken into account with priority. In simulations the interference power is reduced by $30 \mathrm{~dB}$, but also the target corresponding to the interferer is suppressed. The measurements are affected by weaker interference power and a detection of the interferer is still possible after beamforming. 


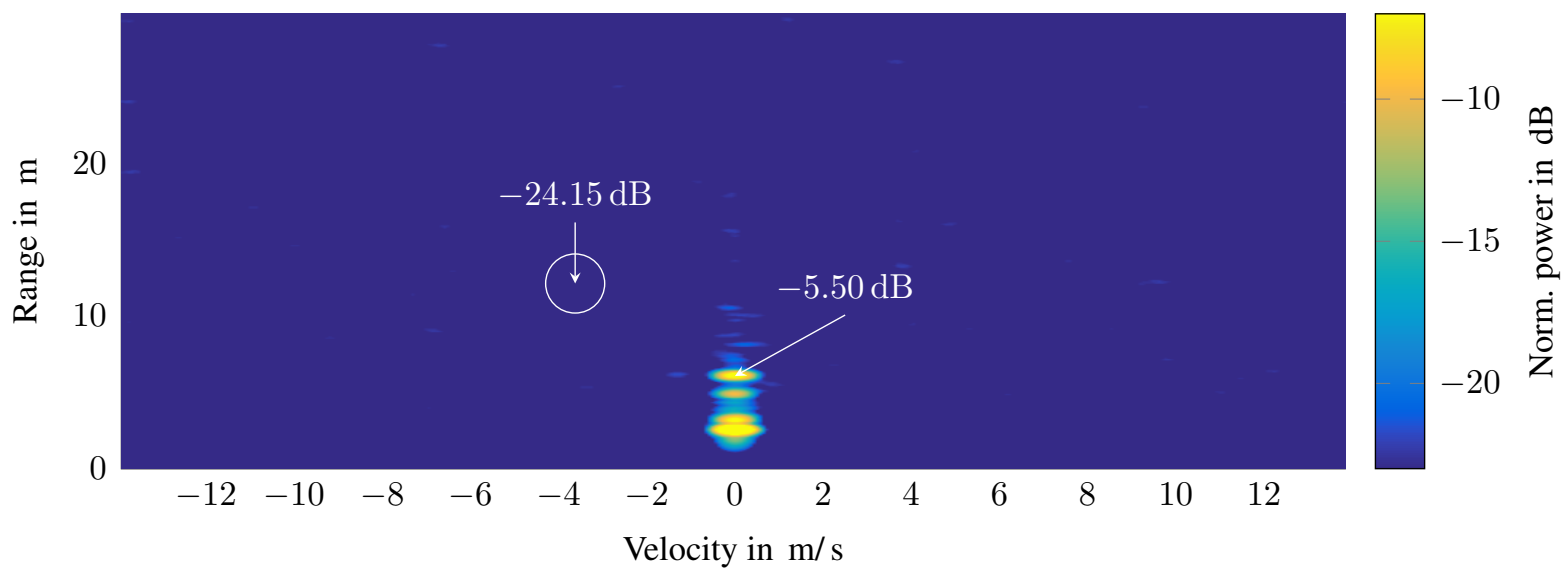

(a) Interference-free range-Doppler spectrum after non-coherent integration.

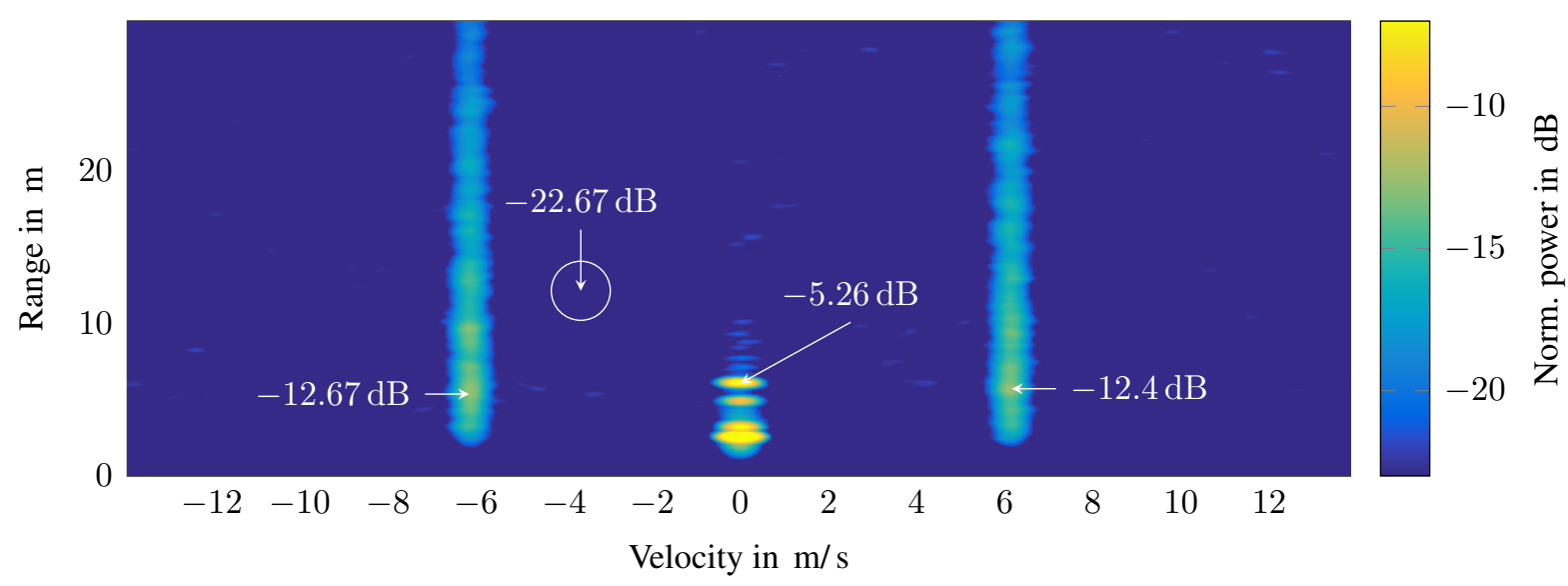

(b) Interfered range-Doppler spectrum after non-coherent integration.

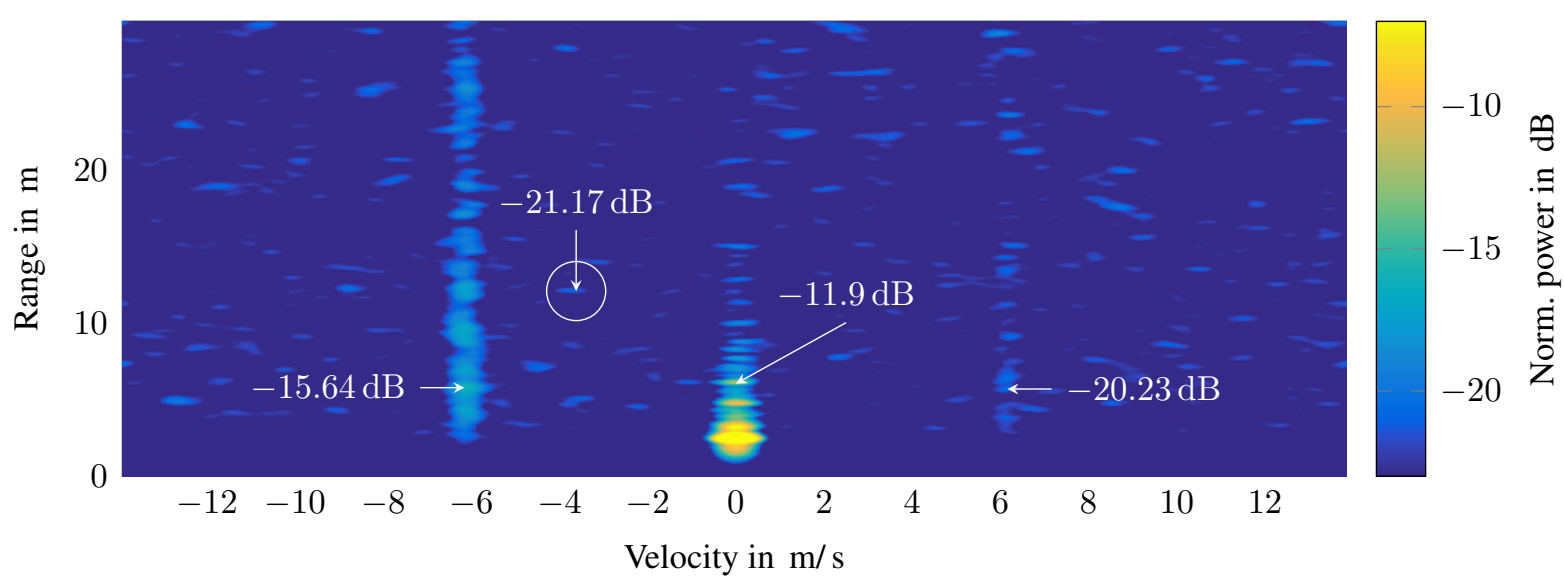

(c) Output of the adaptive digital beamformer. The major part of the interference energy is suppressed.

Figure 8: The measurement shows the range-Doppler spectrum of interference-free signal, interfered signal, and ADBF output. The plots are normalized to the maximum of the interference-free curve. 


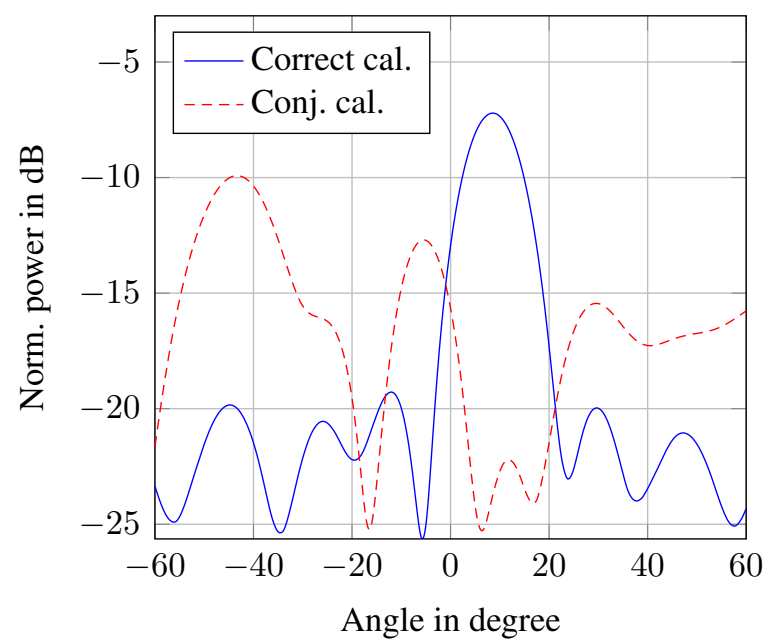

(a) Angle estimation for the velocity cell of $6.16 \frac{\mathrm{m}}{\mathrm{s}}$.

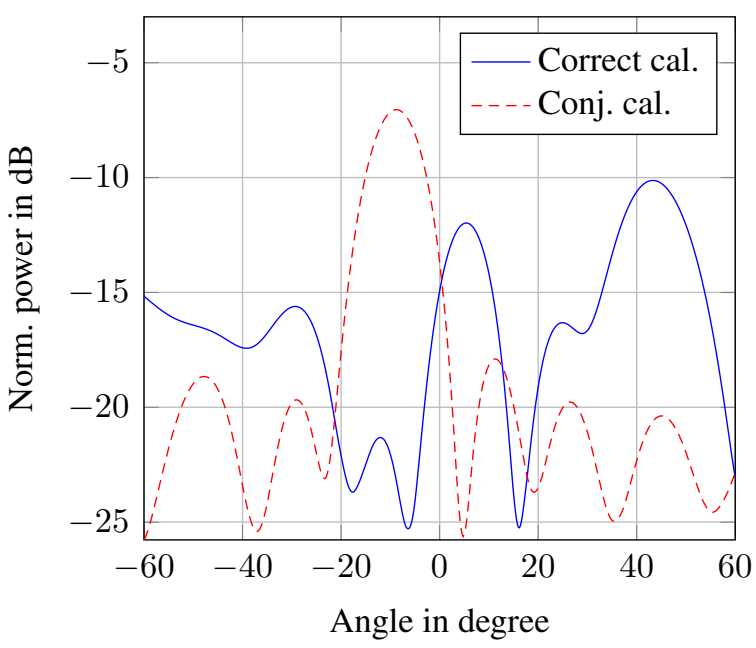

(b) Angle estimation for the velocity cell of $-6.16 \frac{\mathrm{m}}{\mathrm{s}}$.

Figure 9: Comparison of the DoA estimation in case of the correct and the complex conjugate calibration.

\section{References}

[1] D. Oprisan and H. Rohling, "Analysis of Mutual Interference between Automotive Radar Systems," in International Radar Symposium (IRS), 2005, Berlin.

[2] T. Schipper, M. Harter, T. Mahler, O. Kern, and T. Zwick, "Discussion of the operating range of frequency modulated radars in the presence of interference," International Journal of Microwave and Wireless Technologies, vol. 6, pp. 371-378, Jun. 2014.

[3] T. Schipper, T. Mahler, M. Harter, L. Reichardt, and T. Zwick, "An Estimation of the Operating Range for Frequency Modulated Radars in the Presence of Interference," in European Radar Conference (EuRAD), Oct. 2013, pp. 227-230.

[4] B. D. V. Veen and K. M. Buckley, "Beamforming: a versatile approach to spatial filtering," IEEE ASSP Mag., vol. 5, no. 2, pp. 4-24, April 1988.

[5] B. Widrow, P. E. Mantey, L. J. Griffiths, and B. B. Goode, “Adaptive antenna systems,” Proc. IEEE, vol. 55, no. 12, pp. 2143-2159, Dec. 1967.

[6] B. Widrow and S. Stearns, Adaptive signal processing. Englewood Cliffs, N.J.: Prentice-Hall, 1985.

[7] O. Sophocles, Optimum signal processing: an introd., 2nd ed. New York [u.a.]: Macmillan, 1988.

[8] V. Winkler, "Range Doppler Detection for automotive FMCW Radars," in European Microwave Conference, Oct. 2007, pp. 1445-1448.

[9] C. Fischer, M. Goppelt, H.-L. Blöcher, and J. Dickmann, "Minimizing interference in automotive radar using digital beamforming," Advances in Radio Science, vol. 9, pp. 45-48, 2011.

[10] M. Barjenbruch, D. Kellner, K. Dietmayer, J. Klappstein, and J. Dickmann, “A Method for Interference Cancellation in Automotive Radar," in IEEE MTT-S International Conference on Microwaves for Intelligent Mobility (ICMIM), April 2015, pp. 1-4.

[11] C. Fischer, H.-L. Blöcher, J. Dickmann, and W. Menzel, "Robust Detection and Mitigation of Mutual Interference in Automotive Radar," in 16th Int. Radar Symposium (IRS), Jun. 2015.

[12] J. Bechter, M. Rameez, and C. Waldschmidt, "Analytical and Experimental Investigations on Mitigation of Interference in a DBF MIMO Radar," IEEE Trans. Microw. Theory Techn., vol. 65, no. 5, pp. 1727-1734, May 2017. 